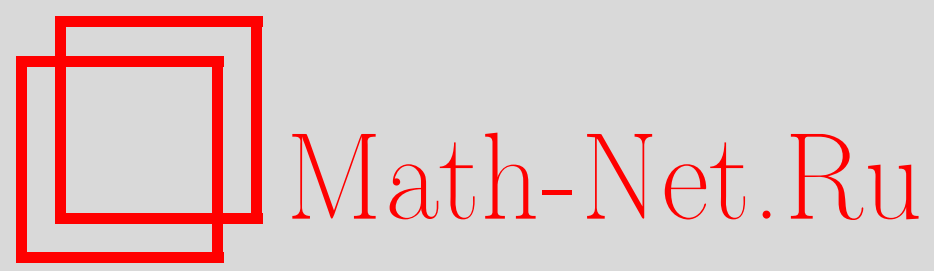

В. И. Заботин, П. А. Чернышевский, Две модификации обобщенного метода Пиявского поиска глобального минимума непрерывной на отрезке функции и их сходимость, Вестник ТвГУ. Серия: Прикладная математика, 2021, выпуск $3,70-85$

DOI: https://doi.org/10.26456/vtpmk624

Использование Общероссийского математического портала Math-Net.Ru подразумевает, что вы прочитали и согласны с пользовательским соглашением

http://www.mathnet.ru/rus/agreement

Параметры загрузки:

IP : 54.162 .27 .143

26 апреля 2023 г., $17: 44: 36$ 


\section{СИСТЕМНЫЙ АНАЛИЗ, УПРАВЛЕНИЕ И ОБРАБОТКА ИНФОРМАЦИИ}

УДК 519.658.4

\section{ДВЕ МОДИФИКАЦИИ ОБОБЩЕННОГО МЕТОДА ПИЯВСКОГО ПОИСКА ГЛОБАЛЬНОГО МИНИМУМА НЕПРЕРЫВНОЙ НА ОТРЕЗКЕ ФУНКЦИИ И ИХ СХОДИМОСТЬ}

Заботин В.И., Чернышевский П.А.

КНИТУ КАИ имени А.Н. Туполева, г. Казань

Поступила в редакцию 0\%.08.2021, после переработки 01.09.2021.

В работах R.J. Vanderbei доказано, что непрерывная на выпуклом компактном множестве функция обладает свойством $\varepsilon$-липшицевости, обобщающим классическое понятие липшицевости. На основе этого свойства R.J. Vanderbei предложено одно обобщение метода Пиявского поиска глобального минимума непрерывной на отрезке функции. В данной работе предлагаются одна модификация этого метода для положительной $\varepsilon$-константы и одна модификация для положительной $\varepsilon$ константы и условия останова, не зависящего от выбора $\varepsilon$. Доказана сходимость предлагаемых алгоритмов, приведены результаты численных экспериментов на основе применения разработанной программы. Данные методы могут быть применены для оптимизации любых непрерывных на отрезке функций, например, при решении некоторых обратных задачах баллистики и в экономике в прямых задачах потребительского выбора маршаллианского типа с переменными ценами благ и с непрерывной функцией полезности.

Ключевые слова: $\varepsilon$-липшицевость, непрерывная функция, глобальная оптимизация, сходимость.

Вестник ТвГУ. Серия: Прикладная математика. 2021. № 3. С. 70-85. https://doi.org/10.26456/vtpmk624

\section{Введение}

Задача поиска глобального экстремума функции на заданном множестве достаточно часто возникает при решении разного рода прикладных задач. Одним из свойств, позволяющим строить численные методы для решения задач глобальной оптимизации, является липшицевость. К настоящему времени предложены и исследованы алгоритмы для одномерного случая [1-5], а также разработаны достаточно эффективные вычислительные схемы для функций многих переменных [6]. Однако, практическая реализация этих методов предполагает априорное

(C) Заботин В.И., Чернышевский П.А., 2021 
знание оценки постоянной Липшица. Последняя, в свою очередь, находится либо аналитически, например, с помощью градиента, или вычисляется непосредственно в ходе работы метода при допущении, что целевая функция липшицева на области поиска.

Тем не менее, для практики представляет также интерес поиск глобального экстремума непрерывной целевой функции без предположения её дифференцируемости. Например, при решении задач нахождения проекции заданной точки на поверхность уровня непрерывной на выпуклом компакте функции [7-11] необходимо отыскивать минимум функции, непрерывной на сфере. Однако непрерывность в общем случае не гарантирует существования константы Липшица, как например, у функций $f(x)=\sqrt{|x|}$ или $f(x)=\arcsin x$. Более того, хорошо известны примеры непрерывных нигде не дифференцируемых на заданном отрезке функций $[12$, стр. 52].

Для построения методов оптимизации непрерывных, но не обязательно липшицевых, функций можно воспользоваться понятием $\varepsilon$-липшицевости, введенным Vanderbei в [13] - функция $f(x)$, определенная на выпуклом множестве $A \subset \mathbb{R}^{n}$, называется $\varepsilon$-липшицевой, если

$$
\forall \varepsilon>0 \exists L(\varepsilon)<+\infty \forall x, y \in A:|f(x)-f(y)| \leq L(\varepsilon)\|x-y\|+\varepsilon .
$$

В той же работе доказана теорема, что если $A$ - выпуклый компакт, то всякая функция будет непрерывна на нем тогда и только тогда, когда для неё на $A$ выполняется свойство (1).

Условие (1) позволяет обобщить некоторые методы оптимизации с липшицевого случая на случай $\varepsilon$-липшицевой функции. Так, все в той же статье [13] предлагается обобщение метода Пиявского поиска глобального минимума функции на отрезке, а в работе [14] предложено обобщение метода Евтушенко. Данные методы требуют по аналогии с «липшицевыми» алгоритмами априорного знания зависимости величины $L(\varepsilon)$ от заданного $\varepsilon>0$. В работах [13-15] в явной форме подобные зависимости получены для функций $f(x)=x^{\alpha}, x \in[0 ; b], 0<\alpha<1$; $f(x)=\ln ^{-1}(x), x \in(0 ; a]$ и $f(x)=\arcsin x$ на $[-1 ; 1]$. Один из возможных численных методов нахождения величины $L(\varepsilon)$ по заданному $\varepsilon>0$ для функций многих переменных, определенных на $n$-мерном брусе, рассмотрен в работе [16].

Как уже отмечалось выше, для липшицевых функций существуют алгоритмы минимизации, не требующие предварительного знания оценки константы Липшица и вычисляющие некоторое приближения этой оценки в ходе вычислений. Одна

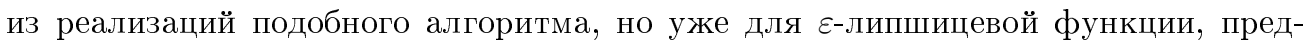
ставлена в работе [17], где был в некотором смысле обобщен метод Стронгина. В той же работе показано, что простой перенос идеи метода Пиявского на случай непрерывной функции может привести к ситуациям, когда на очередном шаге алгоритма невозможно найти новую поисковую точку. Поскольку в оригинальной работе [13] такая проблема не была исследована, возникает необходимость в изучении сходимости алгоритма Vanderbei и в построении такой его модификации, при которой всегда возможно нахождения новой итерационной точки на любом шаге метода. Данная работа и посвящена описанию и обоснованию двух возможных модификаций упомянутого алгоритма.

Отметим, что данные методы можно использовать в прикладных задачах, когда известно лишь только, что целевая функция непрерывна, как например, в 
некоторых обратных задачах баллистики [15] и в экономике при решении задачи потребительского выбора маршаллианского типа с переменными ценами благ и с непрерывной функцией полезности.

\section{1 Описание алгоритмов и их обоснование}

Предлагаемые далее методы будет сформулированы в предположении, что величина $\varepsilon>0$ и функция $f$ согласованы на отрезке $[a ; b]$, то есть существуют такие $x_{0}, y_{0} \in[a ; b]$, что $0<\varepsilon<\left|f\left(x_{0}\right)-f\left(y_{0}\right)\right|$. Данное условие введено в [16], чтобы гарантировать положительность минимальной оценки $l(\varepsilon)=\inf \{L(\varepsilon)\}$ для множества $\{L(\varepsilon)\}$ всех констант $L(\varepsilon)$ при фиксированном $\varepsilon>0$. В противном случае положительность $l(\varepsilon)$ ниоткуда не следует. Там же доказывается, что для согласованных на множестве $A$ функции $f$ и числа $\varepsilon>0$ оценка $l(\varepsilon)$ достижима и $l(\varepsilon)>0$.

Теперь изложим шаги модификации метода Vanderbei для случая положительной оценки $l(\varepsilon)$.

\section{Алгоритм А.}

Вход:

1) функция $f(x)$, непрерывная на отрезке $[a ; b]$;

2 ) величина $\varepsilon>0$, согласованная с функцией $f(x)$ на отрезке $[a ; b]$;

$3)$ величина $l(\varepsilon)$ или её верхняя оценка, которая может быть получена, например, алгоритмами из $[16,17] ;$

4) точность $\delta>\varepsilon$.

\section{Выход:}

1) приближенное с точностью до $\delta$ значение $f\left(v_{*}\right)$ величины $f_{*}=\min _{x \in[a ; b]} f(x)$;

2 ) точка $v_{*}$ отрезка $[a ; b]$, в которой достигается указанное приближенное значение.

Шаг 0. Выбрать точки $u_{0}=v_{0}=a$ и $u_{1}=v_{1}=b$. Аналогично [13] положить первую поисковую точку $v_{*}=a$, если $f(a)<f(b)$, иначе $v_{*}=b$. Положить $k=1$.

Шаг 1. Для каждого отрезка $\left[u_{i-1} ; u_{i}\right]$ вычислить характеристику

$$
R_{k}(i, \varepsilon)=\frac{f\left(u_{i}\right)+f\left(u_{i-1}\right)}{2}-\frac{l(\varepsilon)\left(u_{i}-u_{i-1}\right)}{2}-\varepsilon
$$

аналогичную величине $u\left(y_{l}, y_{r}\right)$ из работы [13]. Далее определить минимальный номер $s$ такой, что

$$
R_{k}(s, \varepsilon)=\min _{1 \leq i \leq k} R_{k}(i, \varepsilon), R_{k}(i, \varepsilon)>R_{k}(s, \varepsilon), i=1, \ldots, s-1 .
$$

Шаг 2. Проверить условие останова

$$
f\left(v_{*}\right)-R_{k}(s, \varepsilon)<\delta .
$$

Если оно выполняется, то завершить алгоритм, полагая приближенно $f_{*}=f\left(v_{*}\right)$, иначе перейти к шагу 3 .

Шаг 3. Вычислить очередную точку

$$
v_{k+1}=\frac{\left(u_{s}+u_{s-1}\right)}{2}-\frac{\left(f\left(u_{s}\right)-f\left(u_{s-1}\right)\right)}{2 l(\varepsilon)} .
$$


Шаг 4. Если $f\left(v_{k+1}\right)<f\left(v_{*}\right)$, то положить $v_{*}=v_{k+1}$, иначе $v_{*}$ оставить прежним.

Шаг 5. Перенумеровать точки $u_{0}, \ldots, u_{k}, v_{k+1}$ в порядке их возрастания. Очевидно, что наименьшая из полученных точек равна $a$, а наибольшая $-b$. Положить $k=k+1$ и перейти к шагу 1 . Алгоритм описан.

Для доказательства сходимости алгоритма выше введем в рассмотрение несколько вспомогательных функций. Определим кусочно-линейную функцию $g\left(x, x_{0}, \varepsilon\right)=f\left(x_{0}\right)-l(\varepsilon)\left|x-x_{0}\right|-\varepsilon$ для фиксированных точки $x_{0} \in[a ; b]$ и величины $\varepsilon>0$ и покажем, что выполняется неравенство

$$
g\left(x, x_{0}, \varepsilon\right) \leq f(x) \forall x \in[a ; b] .
$$

Действительно,

$$
\begin{aligned}
& f(x)-g\left(x, x_{0}, \varepsilon\right)=f(x)-f\left(x_{0}\right)+l(\varepsilon)\left|x-x_{0}\right|+\varepsilon \geq \\
& \geq l(\varepsilon)\left|x-x_{0}\right|+\varepsilon-\left|f(x)-f\left(x_{0}\right)\right| \geq(\text { комментарий: условие }(1)) \geq 0 .
\end{aligned}
$$

Далее, введем в рассмотрение по аналогии с [18, стр. 29] функцию

$$
g(x, \varepsilon)=\max \left\{g\left(x, u_{n}, \varepsilon\right), p_{n-1}(x, \varepsilon)\right\}=\max _{0 \leq i \leq n} g\left(x, u_{i}, \varepsilon\right) .
$$

Считаем, как и раньше, что $f_{*}=\min _{x \in[a ; b]} f(x)$, а $x_{*}$ - точка, в которой достигается указанный глобальный минимум. Будем считать также, что выполнено условие согласованности, описанное выше.

Покажем теперь, что алгоритм находит значение аргумента, при котором значение функции отклоняется от глобального минимума меньше, чем на $\delta>\varepsilon$.

Предложение 1. Пусть $f(x)$ непрерывна на отрезке $[a ; b]$ и удовлетворлет условию (1) $c l(\varepsilon)>0$ при заданном $\varepsilon>0$. Тогда алгоритм $A$ завершается за конечное число иагов, причем условие останова (4) выполняется тогда и только тогда, когда для $v_{*}$ имеет место оценка

$$
0 \leq f\left(v_{*}\right)-f_{*}<\delta .
$$

Доказательство. В работе [17] показано, что при вычислении очередной поисковой точки $v_{k+1}$ формулой (5) выполнение неравенства

$$
u_{s-1}<v_{k+1}<u_{s}
$$

не гарантируется. Таким образом, возможны две ситуации при построении последовательности $\left\{v_{k}\right\}$ с помощью алгоритма А. Ситуация первая: условие (10) выполняется на каждой итерации метода при выполнении шага 3 и все точки $v_{k+1}$ принадлежат своим интервалам поиска $\left(u_{s-1} ; u_{s}\right)$ и процесс построения $\left\{v_{k}\right\}$ становится бесконечным.Ситуация вторая: на некотором шаге неравенство (10) нарушается, и поисковая точка $v_{k+1}$ оказывается вне указанного интервала, что делает невозможным дальнейшее построение последовательности $\left\{v_{k}\right\}$.

В первом случае дальнейшие рассуждения можно провести, пользуясь идеей доказательства теоремы 3 из [18, стр. 30-31]. Из построения $v_{k+1}$ как точки, для 
которой $p_{k}\left(v_{k+1}, \varepsilon\right)=R_{k}(s, \varepsilon)$ (при условии, что точки $v_{k+1}$ на каждом шаге принадлежат своим интервалам), имеем

$$
p_{k}\left(v_{k+1}, \varepsilon\right)=\min _{x \in[a ; b]} p_{k}(x, \varepsilon)
$$

Легко видеть, что

$$
p_{n-1}(x, \varepsilon)=\max _{0 \leq i \leq n-1} g\left(x, u_{i}, \varepsilon\right) \leq \max _{0 \leq i \leq n} g\left(x, u_{i}, \varepsilon\right)=p_{n}(x, \varepsilon), \quad \forall x \in[a ; b] .
$$

Далее из $(6)$ и $l(\varepsilon)>0$ следует

$$
p_{k}(x, \varepsilon) \leq f(x), \quad x \in[a ; b], k=0,1, \ldots
$$

Тогда имеем

$$
\begin{aligned}
& p_{k-1}\left(v_{k}, \varepsilon\right)=\min _{x \in[a ; b]} p_{k-1}(x, \varepsilon) \leq p_{k-1}\left(v_{k+1}, \varepsilon\right) \leq \\
& \leq p_{k}\left(v_{k+1}, \varepsilon\right)=\min _{x \in[a ; b]} p_{k}(x, \varepsilon) \leq p_{k}\left(x_{*}, \varepsilon\right) \leq f_{*},
\end{aligned}
$$

а значит последовательность $\left\{p_{k}\left(v_{k+1}, \varepsilon\right)\right\}$ монотонно возрастает и ограничена сверху, то есть существует предел $\lim _{k \rightarrow \infty} p_{k}\left(v_{k+1}, \varepsilon\right)=p_{*} \leq f_{*}$.

Обозначим через $v$ какую-либо предельную точку (очевидно существующую) последовательности $\left\{v_{k}\right\}$ и через $\left\{v_{k_{m}}\right\}$ сходящуюся к $v\left(\right.$ при $\left.k_{m} \rightarrow+\infty\right)$ подпоследовательность. Так как условие (10) выполняется всегда, то верно равенство $p_{k}\left(v_{i}, \varepsilon\right)=f\left(v_{i}\right)-\varepsilon$. Далее

$$
\begin{aligned}
& 0 \leq p_{k}\left(v_{i}, \varepsilon\right)-\min _{x \in[a ; b]} p_{k}(x, \varepsilon)=f\left(v_{i}\right)-\varepsilon-p_{k}\left(v_{k+1}, \varepsilon\right)= \\
& =p_{k}\left(v_{i}, \varepsilon\right)-p_{k}\left(v_{k+1}, \varepsilon\right) \leq l(\varepsilon)\left|v_{i}-v_{k+1}\right|
\end{aligned}
$$

при любом $k$ и $i=0,1, \ldots, k$. Последнее неравенство справедливо в силу того, что функция $p_{k}(x, \varepsilon)$ липшицева на отрезке $[a ; b]$ с константой $l(\varepsilon)>0$ по построению. Принимая, как и в [12, стр. 30-31], $k=k_{m}-1, i=k_{m-1} \leq k_{m}-1$, получаем

$$
0 \leq f\left(v_{k_{m-1}}\right)-\varepsilon-p_{k_{m}-1}\left(v_{k_{m}}\right) \leq l(\varepsilon)\left|v_{k_{m-1}}-v_{k_{m}}\right| .
$$

Переходя к пределу при $k_{m} \rightarrow \infty$, имеем с учетом непрерывности $f$

$$
0 \leq f(v)-p_{*} \leq \varepsilon
$$

из чего, принимая во внимание, что $p_{*} \leq f_{*} \leq f(v)$, получаем $0 \leq f(v)-f_{*} \leq \varepsilon$. Так как $f\left(v_{k_{m-1}}\right) \rightarrow f(v)$ при $k_{m} \rightarrow \infty$, то для заданного $\varepsilon^{\prime}>0$ такого, что $\varepsilon+\varepsilon^{\prime}<\delta$, существует номер $t$, что $\left|f\left(v_{t}\right)-f(v)\right|<\varepsilon^{\prime}$. Тогда

$$
0 \leq f\left(v_{t}\right)-f_{*} \leq \varepsilon+\varepsilon^{\prime}<\delta .
$$

Пусть точка $v_{t}$ получена на некотором шаге $k$ работы алгоритма. В силу $(13)$, (18) и из построения $R_{k}(s, \varepsilon)$ согласно (3) имеем $f\left(v_{t}\right)-R_{k}(s, \varepsilon)<\delta$. Далее заметим, что $v_{*}$ выбирается как $\arg \min _{0 \leq i \leq k} f\left(v_{i}\right)$, а значит выполнится неравенство $f\left(v_{*}\right)-R_{k}(s, \varepsilon)<\delta$, то есть сработает условие останова (4) и алгоритм завершит 
работу. Кроме того, из (18) непосредственно следует оценка (9). Ясно также, что выполнение неравенства (9) в силу $(13)$ и определения $R_{k}(s, \varepsilon)(3)$ сразу же влечет за собой (4).

Теперь рассмотрим ситуацию невыполнения неравенства (10) на $k$-ом шаге алгоритма. Предположим, что поисковая точка $v_{k+1}$ расположена вне пределов интервала $\left(u_{s-1} ; u_{s}\right)$, как показано на Рис. 1 , причем $v_{k+1}<u_{s-1}$. Тогда справедливо

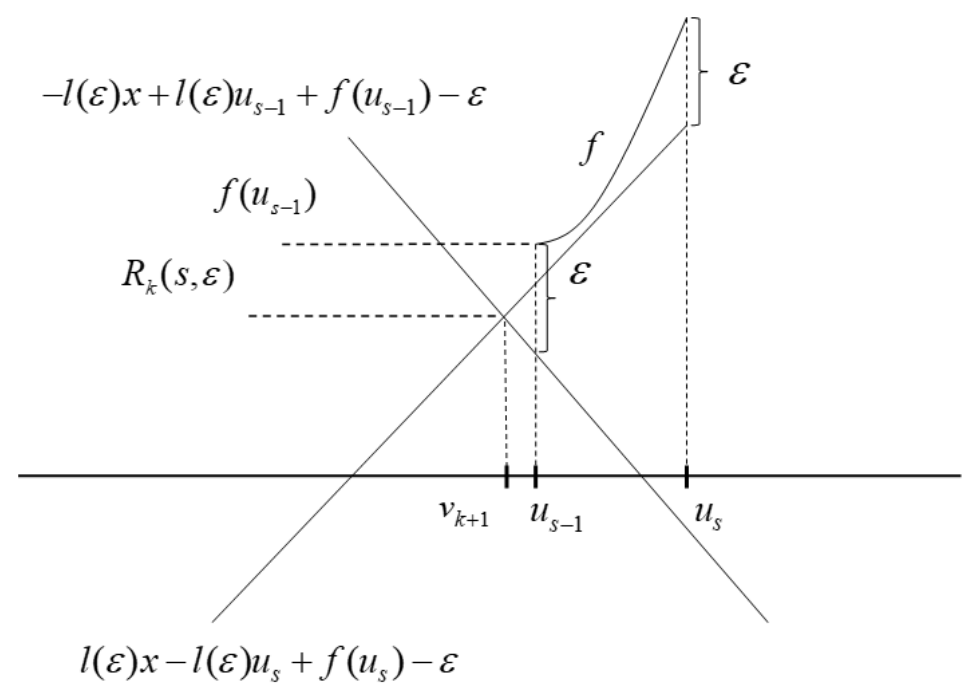

Рис. 1: Графическая интерпретачия случая, когда на $k+1$-ом шаге поисковая точка оказывается вне области поиска.

$$
0 \leq f\left(u_{s-1}\right)-R_{k}(s, \varepsilon) \leq \varepsilon
$$

так как из неравенства (6) и процедуры построения ясно, что если $R_{k}(s, \varepsilon)<f\left(u_{s-1}\right)-\varepsilon$, то точка $v_{k+1}$ будет принадлежать отрезку $\left[u_{s-1} ; u_{s}\right]$, а если $R_{k}(s, \varepsilon)>f\left(u_{s-1}\right)$, то значение функции $g\left(x, u_{s}, \varepsilon\right)=l(\varepsilon) x-l(\varepsilon) u_{s}+f\left(u_{s}\right)-\varepsilon$ в точке $u_{s-1}$ будет больше $f\left(u_{s-1}\right)$, что невозможно в силу (6). Для случая $v_{k+1}>u_{s}$, рассуждая аналогично, имеем

$$
0 \leq f\left(u_{s}\right)-R_{k}(s, \varepsilon) \leq \varepsilon
$$

Поскольку точка $v_{k+1}$ не попала в поисковый интервал $\left(u_{s-1} ; u_{s}\right)$, то возможны две ситуации: $v_{k+1} \in[a ; b]$ и $v_{k+1} \notin[a ; b]$. Если $v_{k+1} \in[a ; b]$, то, учитывая, что условие (10) не нарушалось до $k$-го шага, имеем $R_{k}(s, \varepsilon)=p_{k}\left(v_{k+1}, \varepsilon\right)=\min _{x \in[a ; b]} p_{k}(x, \varepsilon) \leq f_{*}$. Если же $v_{k+1} \notin[a ; b]$, то из построения $R_{k}(s, \varepsilon)$ как $\min _{1 \leq i \leq k} R_{k}(i, \varepsilon)$ получаем $R_{k}(s, \varepsilon) \leq \min _{x \in[a ; b]} p_{k}(x, \varepsilon) \leq f_{*}$. Тогда из (19) и (20) для случаев $v_{k+1} \in[a ; b]$ и $v_{k+1} \notin[a ; b]$ следует, что

$$
0 \leq f\left(u_{s-1}\right)-f_{*} \leq \varepsilon<\delta,
$$


если $v_{k+1}<u_{s-1}$, и

$$
0 \leq f\left(u_{s}\right)-f_{*} \leq \varepsilon<\delta
$$

когда $v_{k+1}>u_{s}$.

Понятно, что вне зависимости от того, выполнится ли (19), либо же (20), условие останова (4) выполнится, а так как оно проверяется до нахождения новой точки $v_{k+1}$, алгоритм корректно завершит работу. Из характера построения $v_{*}$, а также из (21) и (22), сразу вытекает и оценка (9).

Таким образом, в обеих ситуациях (бесконечношаговый и конечношаговый процесс) алгоритм завершает работу за конечное число шагов, причем одновременно с условием останова (4) выполняется неравенство (9).

В алгоритме из работы [17, стр. 1113] нарушение условия (10) при поиске очередной поисковой точки не приводит к остановке метода за счет ввода шагов 3 и 4 , позволяющих находить итерационную точку строго в поисковом интервале. Применив данный прием к алгоритму А, можно получить модификацию метода, которая в отличие от алгоритма А и алгоритма Vanderbei из [13] дает возможность использовать условие останова, отличное от (4) и не зависящее от величины $\varepsilon>0$. Изложим один из вариантов такой модификации.

\section{Алгоритм Б.}

\section{Вход:}

1) функция $f(x)$, непрерывная на отрезке $[a ; b]$

2) величина $\varepsilon>0$, согласованная с функцией $f(x)$ на отрезке $[a ; b]$;

$3)$ величина $l(\varepsilon)$ или её верхняя оценка, которая может быть получена, например, алгоритмами из $[16,17]$;

4) заданы параметр $\mu>1$ и критерий останова.

\section{Выход:}

1) приближенное значение $f\left(v_{*}\right)$ величины $f_{*}=\min _{x \in[a ; b]} f(x)$;

$2)$ точка $v_{*}$ отрезка $[a ; b]$, в которой достигается указанное приближенное значение.

Шаг 0. Выбрать точки $u_{0}=v_{0}=a$ и $u_{1}=v_{1}=b$. Аналогично [13] положить первую поисковую точку $v_{*}=a$, если $f(a)<f(b)$, иначе $v_{*}=b$. Положить $k=1$.

Шаг 1. Для каждого отрезка $\left[u_{i-1} ; u_{i}\right]$ вычислить характеристику

$$
R_{k}(i, \varepsilon)=\frac{f\left(u_{i}\right)+f\left(u_{i-1}\right)}{2}-\frac{l(\varepsilon)\left(u_{i}-u_{i-1}\right)}{2}-\varepsilon
$$

аналогичную величине $u\left(y_{l}, y_{r}\right)$ из работы [13]. Далее определить минимальный номер $s$ такой, что

$$
R_{k}(s, \varepsilon)=\min _{1 \leq i \leq k} R_{k}(i, \varepsilon), R_{k}(i, \varepsilon)>R_{k}(s, \varepsilon), i=1, \ldots, s-1 .
$$

Шаг 2. Положить $l^{\prime}(\varepsilon)=l(\varepsilon)$.

Шаг 3. Вычислить очередную точку

$$
v_{k+1}=\frac{\left(u_{s}+u_{s-1}\right)}{2}-\frac{\left(f\left(u_{s}\right)-f\left(u_{s-1}\right)\right)}{2 l^{\prime}(\varepsilon)}
$$

и проверить условие 


$$
u_{s-1}<v_{k+1}<u_{s}
$$

Если условие (26) выполнено сразу, то перейти к шагу 4. Если условие (26) не выполняется, то положить $l^{\prime}(\varepsilon)=\mu l^{\prime}(\varepsilon)$ и повторять шаг 3 до тех пор, пока условие (26) не выполнится, что гарантируется увеличением $l^{\prime}(\varepsilon)($ см. $(25))$, после чего перейти к шагу 4.

Шаг 4. Если $f\left(v_{k+1}\right)<f\left(v_{*}\right)$, то положить $v_{*}=v_{k+1}$, иначе $v_{*}$ оставить прежним.

Шаг 5. Проверить заданное условие останова. Если оно выполняется, то завершить работу алгоритма, полагая приближенно $f_{*}=f\left(v_{*}\right)$. Если оно не выполняется, то перенумеровать точки $u_{0}, \ldots, u_{k}, v_{k+1}$ в порядке их возрастания. Очевидно, что наименьшая из полученных точек равна $a$, а наибольшая $-b$. Положить $k=k+1$ и перейти к шагу 1. Алгоритм описан.

Обоснование. Как нетрудно заметить, алгоритм Б отличается от алгоритма А наличием шагов 2 и 3 , что делает его принципиально бесконечношаговым, и в этом случае для останова можно применять любое эвристическое правило, например,

$$
\left\{\begin{array}{l}
\left|f\left(v_{k+1}\right)-f\left(v_{k}\right)\right| \leq \xi \\
\left|v_{k+1}-v_{k}\right| \leq \xi
\end{array}\right.
$$

где значение параметра $\xi>0$ выбирается вычислителем.

\section{2. Численные эксперименты}

Приведем результаты численных экспериментов, проведенных с помощью программного обеспечения, реализующего представленные в работе алгоритмы А и Б. Для алгоритма Б было применено условие останова (27), и установлено значение параметра метода $\mu=2$.

В таблицах используются следующие обозначения: $\delta$ - заданная точность; $\varepsilon$ - параметр метода; $f\left(v_{*}\right)$ - приближенное значение глобального минимума; $v_{*}-$ точка, в котором достигается указанное приближенное значение; $l(\varepsilon)$ - значение оценки минимальной постоянной $l(\varepsilon)$ для заданного $\varepsilon ; f\left(v_{*}\right)-R_{k}(s, \varepsilon)-$ левая часть неравенства (4) из алгоритма $\mathrm{A} ; \Delta_{k} f$ и $\Delta_{k} v$ - левые части условия (27) для алгоритма Б.

Пример 1. Ищется минимум функции

$$
f_{1}(x)=\min \{\sqrt{|x+4|}-1 ; \sqrt{|x+1|}-1.005 ; \sqrt{|x-3|}+0.5\}
$$

из работы [14], в которой поиск глобального минимума для $f_{1}(x)$ производился на различных отрезках с помощью обобщенного метода Евтушенко при известной зависимости величины оценки $l(\varepsilon)$ от значений $\varepsilon>0$. Результаты вычислений, проведенных с помощью алгоритма А и Б, представлены соответственно в Таблицах 1 и 2. Как можно заметить из таблиц, результаты работы методов с хорошей точностью совпадают с полученными в [14]. Для наглядности на Рис. 2 представлен график оптимизируемой функции $f_{1}(x)$. 
Пример 2. В работе [17] с помощью обобщенного метода Стронгина найден глобальный минимум функции

$$
f_{2}(x)=\left\{\begin{array}{l}
-\arcsin (x+2),-3 \leq x \leq-1 \\
\arcsin x,-1 \leq x \leq 0 \\
-\arcsin x, 0 \leq x \leq 0.9
\end{array}\right.
$$

на отрезке $[-3 ; 0.9]$. Результаты расчетов для данной функции показаны в Таблицах 3 и 4, а на Рис. 3 представлен график функции $f_{2}(x)$. Значения оценок $l(\varepsilon)$ для фиксированных $\varepsilon>0$ были взяты из работы [17], где они были вычислены в ходе работы метода. Полученные результаты согласуются с работой [17].

Таблица 1: Результаты вычислений с помощью алгоритма А для функции $f_{1}(x)$

\begin{tabular}{|c|c|c|c|c|c|c|c|}
\hline Отрезок & $\delta$ & $\varepsilon$ & $l(\varepsilon)$ & $v_{*}$ & $f\left(v_{*}\right)$ & Усл.(3) & Число шагов \\
\hline \multirow{6}{*}[-5;5]{} & \multirow{2}{*}{0.1} & 0.05 & 5.0 & -4.002 & -0.945 & 0.096 & 41 \\
\hline & & 0.01 & 25.0 & -3.999 & -0.968 & 0.096 & 191 \\
\hline & \multirow{2}{*}{0.01} & 0.005 & 50.0 & -1.000 & -0.996 & 0.009 & 431 \\
\hline & & 0.001 & 250.0 & -0.999 & -1.000 & 0.009 & 2149 \\
\hline & \multirow{2}{*}{0.001} & 0.0005 & 500.0 & -1.000 & -1.004 & $9 E-4$ & 4353 \\
\hline & & 0.0001 & 2500.0 & -1.000 & -1.004 & $7 E-4$ & 20772 \\
\hline \multirow{6}{*}[-10;10]{} & \multirow{2}{*}{0.1} & 0.05 & 5.0 & -3.993 & -0.918 & 0.094 & 63 \\
\hline & & 0.01 & 25.0 & -4.001 & -0.966 & 0.095 & 271 \\
\hline & \multirow{2}{*}{0.01} & 0.005 & 50.0 & -1.000 & -0.997 & 0.009 & 591 \\
\hline & & 0.001 & 250.0 & -1.000 & -1.001 & 0.009 & 2963 \\
\hline & \multirow{2}{*}{0.001} & 0.0005 & 500.0 & -0.999 & -1.004 & $9 E-4$ & 5985 \\
\hline & & 0.0001 & 2500.0 & -0.999 & -1.004 & $9 E-4$ & 29331 \\
\hline
\end{tabular}

Таблица 2: Результаты вычислений с помощью алгоритма Б для функции $f_{1}(x)$

\begin{tabular}{|l|l|l|l|l|l|l|l|l|}
\hline Отрезок & $\xi$ & $\varepsilon$ & $l(\varepsilon)$ & $v_{*}$ & $f\left(v_{*}\right)$ & $\Delta_{k} f$ & $\Delta_{k} v$ & Шаги \\
\hline \multirow{5}{*}[-5;5]{} & \multirow{2}{*}{0.01} & 0.05 & 5.0 & -1.000 & -0.984 & 0.001 & $4 E-5$ & 52 \\
\cline { 2 - 9 } & \multirow{2}{*}{0.001} & 0.01 & 25.0 & -1.000 & -1.002 & 0.009 & $1 E-4$ & 217 \\
\cline { 2 - 9 } & & 0.005 & 50.0 & -1.000 & -1.003 & $3 E-4$ & $4 E-6$ & 435 \\
\cline { 2 - 9 } & \multirow{2}{*}{0.0001} & 0.005 & 50.0 & -1.000 & -1.003 & $4 E-5$ & $9 E-8$ & 437 \\
\cline { 3 - 9 } & \multirow{2}{*}{0.01} & 0.001 & 250.0 & -1.000 & -1.004 & $8 E-8$ & $4 E-5$ & 2183 \\
\hline \multirow{5}{*}[-10;10]{} & \multirow{2}{*}{0.001} & 0.01 & 25.0 & -1.000 & -0.981 & $7 E-4$ & $3 E-5$ & 71 \\
\cline { 3 - 9 } & & 0.005 & 50.0 & -1.000 & -1.003 & $1 E-4$ & $4 E-7$ & 599 \\
\cline { 2 - 8 } & \multirow{2}{*}{0.0001} & 0.005 & 50.0 & -1.000 & -1.003 & $2 E-5$ & $9 E-8$ & 600 \\
\cline { 3 - 9 } & & 0.001 & 250.0 & -1.000 & -1.004 & $3 E-5$ & $2 E-8$ & 2999 \\
\hline
\end{tabular}


Таблица 3: Результаты вычислений с помощью алгоритма А для функции $f_{2}(x)$

\begin{tabular}{|l|l|l|l|l|l|l|l|}
\hline Отрезок & $\delta$ & $\varepsilon$ & $l(\varepsilon)$ & $v_{*}$ & $f\left(v_{*}\right)$ & Усл. (3) & Число шагов \\
\hline \multirow{5}{*}[-3;0.9]{} & \multirow{2}{*}{0.1} & 0.005 & 193.0 & -0.999 & -1.545 & 0.095 & 513 \\
\cline { 3 - 8 } & \multirow{2}{*}{0.015} & 0.001 & 854.0 & -1.000 & -1.559 & 0.099 & 2239 \\
\cline { 3 - 8 } & \multirow{2}{*}{0.01} & 0.005 & 193.0 & -0.999 & -1.566 & 0.011 & 580 \\
\cline { 3 - 8 } & & 0.005 & 193.0 & -0.999 & -1.566 & 0.008 & 583 \\
\hline
\end{tabular}

Таблица 4: Результаты вычислений с помощью алгоритма Б для функции $f_{2}(x)$

\begin{tabular}{|c|c|c|c|c|c|c|c|c|}
\hline Отрезок & $\xi$ & $\varepsilon$ & $l(\varepsilon)$ & $v_{*}$ & $f\left(v_{*}\right)$ & $\Delta_{k} f$ & $\Delta_{k} v$ & Шаги \\
\hline \multirow{6}{*}[-3;0.9]{} & \multirow{2}{*}{0.01} & 0.005 & 193.0 & -1.000 & -1.560 & 0.0065 & $2 E-4$ & 559 \\
\hline & & 0.001 & 854.0 & -0.999 & -1.553 & 0.009 & 0.007 & 637 \\
\hline & \multirow{2}{*}{0.001} & 0.005 & 193.0 & -0.999 & -1.570 & $3 E-4$ & $5 E-7$ & 591 \\
\hline & & 0.001 & 854.0 & -1.000 & -1.569 & $6 E-4$ & $7 E-6$ & 2561 \\
\hline & \multirow{2}{*}{0.0001} & 0.005 & 193.0 & -0.999 & -1.570 & $3 E-4$ & $5 E-7$ & 591 \\
\hline & & 0.001 & 854.0 & -0.999 & -1.570 & $1 E-5$ & $3 E-9$ & 2582 \\
\hline
\end{tabular}

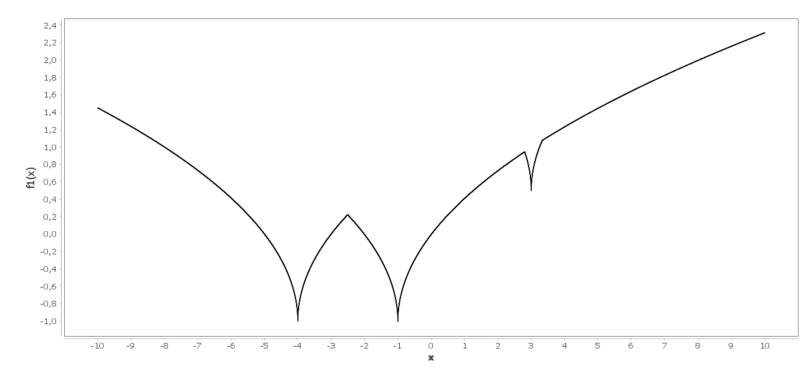

Pис. 2: График функиии $f_{1}(x)$

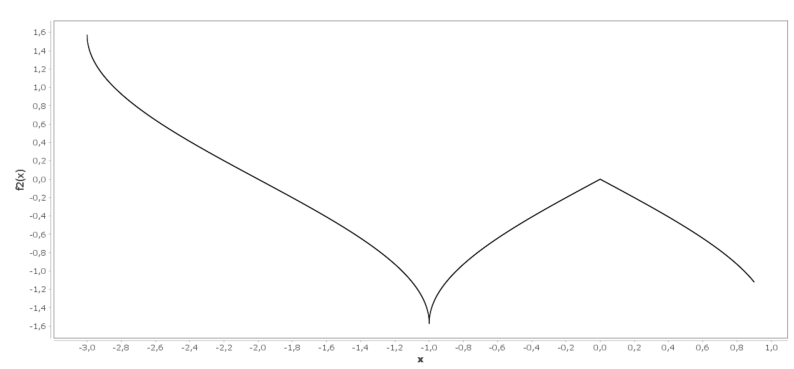

Pис. 3: График функиии $f_{2}(x)$ 


\section{Заключение}

В данной работе предложены две модификации обобщенного метода Пиявского поиска глобального минимума непрерывной на отрезке функции на случай положительной $\varepsilon$-постоянной Липшица и для случая произвольного условия останова, не зависящего от выбора $\varepsilon$. Проведено обоснование предлагаемых методов, приведены результаты расчетов указанными методами. Полученные результаты вычислений с хорошей точностью совпадают с известными.

Авторы статьи считают долгом поблагодарить Фаррухшину Т.Р., студента 2-го курса КНИТУ-КАИ, за помощь в проведении численных экспериментов.

\section{Список литературы}

[1] Пиявский С.А. Один алгоритм отыскания абсолютного минимума функций // Теория оптимальных решений. 1967. Т. 2. С. 13-24.

[2] Пиявский С.А. Один алгоритм отыскания абсолютного экстремума функций // Журнал вычислительной математики и математической физики. 1972. T. 12 , № 4. C. $885-896$.

[3] Евтушенко Ю.Г. Численный метод поиска глобального экстремума функции (перебор на неравномерной сетке) // Журнал вычислительной математики и математической физики. 1971. Т. 11, № 6. С. 1390-1403.

[4] Shubert B. A sequential method seeking the global maximum of a function // SIAM Journal on Numerical Analysis. 1972. Vol. 9. Pp. 379-388.

[5] Стронгин Р.Г. Численные методы в многоэкстремальных задачах. М.: Наука, 1978. $240 \mathrm{c}$.

[6] Сергеев Я.Д., Квасов Д.Е. Диагональные методы глобальной оптимизации. М.: Физматлит, 2008. 352 с.

[7] Заботин В.И., Арутюнова Н.К. Два алгоритма отыскания проекции точки на невыпуклое множество в нормированном пространстве // Журнал вычислительной математики и математической физики. 2013. Т. 53, № 3. С. 344-349.

[8] Арутюнова Н.К., Дуллиев А.М., Заботин В.И. Алгоритмы проектирования точки на поверхность уровня непрерывной на компакте функции // Журнал вычислительной математики и математической физики. 2014. Т. 54, № 9. C. $1448-1454$.

[9] Черняев Ю.А. Численный алгоритм решения задачи математического программирования с ограничением в виде гладкой поверхности // Журнал вычислительной математики и математической физики. 2016. Т. 56, № 3. С. 387393.

[10] Черняев Ю.А. Об одном численном алгоритме решения экстремальных задач с предвыпуклыми ограничениями // Журнал вычислительной математики и математической физики. 2003. Т. 43, № 2. С. 169-175. 
[11] Черняев Ю.А. Обобщение метода проекции градиента и метода Ньютона на экстремальные задачи с ограничением в виде гладкой поверхности // Журнал вычислительной математики и математической физики. 2015. Т. 55, № 9. C. $1493-1502$.

[12] Гелбаум Б., Олмстед Дж. Контрпримеры в анализе. М.: Мир, 1967. 251 с.

[13] Vanderbei R.J. Extension of Piyavskii's Algorithm to Continuous Global Optimization // Journal of Global Optimization. 1999. Vol. 14. Pp. 205-216.

[14] Арутюнова Н.К. Метод Евтушенко поиска глобального минимума $\varepsilon-$ липшицевой функции и его приложения // Вестник КГТУ им. А.Н. Туполева. 2013. № 2. C. 154-157.

[15] Arutyunova N.K., Dulliev A.M., Zabotin V.I. Models and methods for three external ballistics inverse problems // Vestnik Yuzhno-Uralskogo gosudarstvennogo universiteta. Seriya: Matematicheskoe modelirovanie i programmirovanie. 2017. Vol. 10, № 4. Pp. 78-91.

[16] Заботин В.И., Чернышевский П.А. Алгоритм вычисления минимальной оценки $\varepsilon$-постоянной Липшица непрерывной функции // Вестник КГТУ им. А.Н. Туполева. 2018. № 2. С. 127-132.

[17] Zabotin V.I., Chernyshevskij P.A. Extension of Strongin's Global Optimization Algorithm to a Function Continuous on a Compact Interval // Computer Research and Modeling. 2019. Vol. 11. Pp. 1111-1119.

[18] Васильев Ф.П. Численные методы решения экстремальных задач. Учеб. пособие для вузов. 2-е изд., перераб. и доп.. М.: Наука, 1988. 552 с.

\section{Образец цитирования}

Заботин В.И., Чернышевский П.А. Две модификации обобщенного метода Пиявского поиска глобального минимума непрерывной на отрезке функции и их сходимость // Вестник ТвГУ. Серия: Прикладная математика. 2021. № 3. С.70-85. https://doi.org/10.26456/vtpmk624 


\section{Сведения об авторах}

\section{1. Заботин Владислав Иванович}

профессор кафедры прикладной математики и информатики им. Ю.В. Кожевникова Казанского национального исследовательского технического университета им. А.Н. Туполева КАИ.

Россия, 420015, Республика Татарстан, г. Казань, улица Большал Краснал, д. 55, 7-е учебное здание КНИТУ-КАИ. E-mail: v.zabotin@rambler.ru

\section{2. Чернышевский Павел Андреевич}

ассистент кафедры прикладной математики и информатики им. Ю.В. Кожевникова Казанского национального исследовательского технического университета им. А.Н. Туполева КАИ.

Россия, 420015, Республика Татарстан, г. Казанъ, улица Большал Красная, д. 55, 7-е учебное здание КНИТУ-КАИ. E-mail: pavelcomm@mail.ru 


\title{
TWO MODIFICATIONS OF EXTENSION OF PIYAVSKII'S GLOBAL OPTIMIZATION ALGORITHM TO A FUNCTION CONTINUOUS ON A COMPACT INTERVAL AND ITS CONVERGENCE
}

\author{
Zabotin Vladislav Ivanovich \\ Professor at Applied Mathematics and Informatics department, \\ Kazan National Research Technical University named after A. N. Tupolev - KAI \\ Russia, 420015, Tatarstan, Kazan, 55 Bolshaya Krasnaya Str., Academic Building \\ No. 7 . \\ E-mail:v.zabotin@rambler.ru

\section{Chernyshevskij Pavel Andreevich} \\ Teaching assistant at Applied Mathematics and Informatics department, \\ Kazan National Research Technical University named after A. N. Tupolev - KAI \\ Russia, 420015, Tatarstan, Kazan, 55 Bolshaya Krasnaya Str., Academic Building \\ No. 7. \\ E-mail: pavelcomm@mail.ru
}

\begin{abstract}
Received 0\%.08.2021, revised 01.09.2021.
R.J. Vanderbei in his works proves that any continuous on a compact set function has the $\varepsilon$-Lipschitz property which extends conventional Lipschitz continuity. Based on this feature Vanderbei proposed one extension of Piyavskii's global optimization algorithm to the continuous function case. In this paper we propose one modification of the Vanderbei's algorithm for a positive $\varepsilon$-constant and another modification for a positive $\varepsilon$-constant and $\varepsilon$ value independent termination condition. We prove proposed methods convergence and perform several computational experiments with designed software for known test functions.
\end{abstract}

Keywords: $\varepsilon$-Lipschitz continuity, continuous function, global optimization, algorithm convergence.

\section{Citation}

Zabotin V.I., Chernyshevskij P.A., "Two modifications of extension of piyavskii's global optimization algorithm to a function continuous on a compact interval and its convergence", Vestnik TvGU. Seriya: Prikladnaya Matematika /Herald of Tver State University. Series: Applied Mathematics], 2021, № 3, 70-85 (in Russian). https://doi.org/10.26456/vtpmk624

\section{References}

[1] Piyavskij S.A., "An algorithm for finding the absolute minimum of a function", Teoriya optimalnykh reshenij [Theory of Optimal Solutions], 2 (1967), 13-24 (in Russian). 
[2] Piyavskij S.A., "An algorithm for finding the absolute extremum of a function", USSR Computational Mathematics and Mathematical Physics, 12:4 (1972), 57-67.

[3] Yevtushenko Yu.G., "Numerical methods for finding global extrema (Case of a non-uniform mesh)", USSR Computational Mathematics and Mathematical Physics, 11:6 (1971), 38-54.

[4] Shubert B., "A sequential method seeking the global maximum of a function", SIAM Journal on Numerical Analysis, 9 (1972), 379-388.

[5] Strongin R.G., Numerical methods in multiextremal problems, Nauka Publ., Moscow, 1978 (in Russian), 240 pp.

[6] Sergeev Ya.D., Kvasov D.E., Diagonal methods of global optimization, Fizmatlit Publ., Moscow, 2008 (in Russian), 352 pp.

[7] Zabotin V.I., Arutyunova N.K., "Two algorithms for finding point projection on nonconvex set in normalized space", Zhurnal vychislitelnoj matematiki $i$ matematicheskoj fiziki [Journal of Computational Mathematics and Mathematical Physics], 53:3 (2013), 344-349 (in Russian).

[8] Arutyunova N.K., Dulliev A.M., Zabotin V.I., "Algorithms for projecting a point onto a level surface of a continuous function on a compact set", Computational Mathematics and Mathematical Physics, 54:9 (2014), 1395-1401.

[9] Chernyaev Yu.A., "Numerical algorithm for solving mathematical programming problems with a smooth surface as a constraint", Computational Mathematics and Mathematical Physics, 56:3 (2016), 376-381.

[10] Chernyaev Yu.A., "On a numerical algorithm for optimization problems with preconvex constraints", Computational Mathematics and Mathematical Physics, 43:2 (2003), 162-167.

[11] Chernyaev Yu.A., "An extension of the gradient projection method and Newton's method to extremum problems constrained by a smooth surface", Computational Mathematics and Mathematical Physics, 55:9 (2015), 1451-1460.

[12] Gelbaum B., Olmsted Dzh., Kontrprimery v analize [Counterexamples in analysis], Mir Publ., Moscow, 1967 (in Russian), 251 pp.

[13] Vanderbei R.J., "Extension of Piyavskii's Algorithm to Continuous Global Optimization", Journal of Global Optimization, 14 (1999), 205-216.

[14] Arutyunova N.K., "Yevtushenko's method for finding $\varepsilon$-Lipschitzian function global minimum and its application", Vestnik KGTU im. A.N. Tupoleva [Herald of the KSTU-KAI Named After A.N. Tupolev], 2013, № 2, 154-157 (in Russian).

[15] Arutyunova N.K., Dulliev A.M., Zabotin V.I., "Models and methods for three external ballistics inverse problems", Vestnik Yuzhno-Uralskogo gosudarstvennogo universiteta. Seriya: Matematicheskoe modelirovanie i programmirovanie [Bulletin of the South Ural State University: Series "Mathematical modelling, Programming and Computer software"], 10:4 (2017), 78-91. 
[16] Zabotin V.I., Chernyshevskij P.A., "An algorithm for calculation the minimal estimate of the continuous function Lipschitz $\varepsilon$-constant", Vestnik KGTU im. A.N. Tupoleva [Herald of the KSTU-KAI Named After A.N. Tupolev], 2018, № 2, 127132 (in Russian).

[17] Zabotin V.I., Chernyshevskij P.A., "Extension of Strongin's Global Optimization Algorithm to a Function Continuous on a Compact Interval", Computer Research and Modeling, 11 (2019), 1111-1119.

[18] Vasilev F.P., Chislennye metody resheniya ekstremalnykh zadach, Ucheb. posobie dlya vuzov, 2-e izd., pererab. i dop., Nauka Publ., Moscow, 1988 (in Russian), $552 \mathrm{pp}$. 